\title{
Lumières au pluriel
}

Diffusion des savoirs et projets éducatifs au XVIII ${ }^{\mathrm{e}}$ siècle

\section{Catriona Seth et Rotraud von Kulessa}

\section{(2) OpenEdition}

Journals

Édition électronique

URL : http://journals.openedition.org/aes/2436

DOI : 10.4000/aes.2436

ISSN : 2258-093X

Éditeur

Laboratoire LISAA

Référence électronique

Catriona Seth et Rotraud von Kulessa, «Lumières au pluriel », Arts et Savoirs [En ligne], 13 | 2020, mis en ligne le 01 juin 2020, consulté le 24 septembre 2020. URL : http://journals.openedition.org/aes/ 2436 ; DOI : https://doi.org/10.4000/aes.2436

Ce document a été généré automatiquement le 24 septembre 2020.

Centre de recherche LISAA (Littératures SAvoirs et Arts) 


\section{Lumières au pluriel}

Diffusion des savoirs et projets éducatifs au XVIII siècle

\section{Catriona Seth et Rotraud von Kulessa}

In memoriam Sophie Lefay ${ }^{1}$

Comment pourrait-on, sans la connaissance des langues, sans l'habitude de la lecture, communiquer avec ces hommes qui ne sont plus, et que nous sentons si bien nos amis, nos concitoyens, nos alliés ? Il faut être médiocre de cœur pour se refuser à de si nobles plaisirs. Ceuxlà seulement qui remplissent leur vie de bonnes œuvres peuvent se passer de toute étude :

l'ignorance, dans les hommes oisifs, prouve autant la sécheresse de l'âme que la légèreté de l'esprit. Enfin, il reste encore une chose vraiment belle et morale, dont l'ignorance et la frivolité ne peuvent jouir : c'est l'association de tous les hommes qui pensent, d'un bout de l'Europe à l'autre. Souvent

ils n'ont entre eux aucune relation; ils sont dispersés souvent à de grandes distances l'un de l'autre ; mais quand ils se rencontrent, un mot suffit pour qu'ils se reconnaissent. Ce n'est pas telle religion, telle opinion, tel genre d'étude, c'est le culte de la vérité qui les réunit. Tantôt, comme les mineurs, ils creusent jusqu'au fond de la terre, pour pénétrer, au sein de l'éternelle nuit,

les mystères du monde ténébreux ; tantôt ils étudient les langues de l'Orient, pour y chercher l'histoire primitive de l'homme ; tantôt ils vont à 
Jérusalem pour faire sortir des ruines saintes une étincelle qui ranime la religion et la poésie ; enfin, ils sont vraiment le peuple de Dieu, ces hommes qui ne désespèrent pas encore de la race humaine, et veulent lui conserver l'empire de la pensée. $^{2}$

1 Publiées en 1813 dans De l'Allemagne, œuvre dont le duc de Rovigo, ministre de la Police de Napoléon, avait ordonné la destruction trois ans plus tôt pour un motif clair - «ce livre n'est pas français" - ces lignes sonnent comme un manifeste des Lumières européennes et plurielles. Au cœur du projet qui unit des individus aux convictions diverses, d'origines culturelles multiples et aux sensibilités variables, il y a en effet une volonté réelle de diffusion du savoir. Pour les esprits éclairés, l'éducation et la transmission des idées doivent contribuer à rendre le monde meilleur.

2 Des recherches récentes ont mis en évidence l'importance des réseaux savants - les milieux académiques et la cartographie des échanges scientifiques ont fait l'objet de nombreux travaux, en partie grâce à l'apport de la numérisation. Le projet ENCCRE (Édition numérique collaborative et critique de l'Encyclopédie de Diderot, de D'Alembert et de Jaucourt ${ }^{3}$ ) part d'un exemplaire de l'Encyclopédie, œuvre, comme le rappelle la page de titre, d'une « société de Gens de Lettres »-, pour analyser les savoirs présentés. Electronic Enlightenment, un corpus de correspondances des Lumières continuellement en expansion ${ }^{4}$, s'enorgueillit, au 13 mai 2020, de donner accès au " premier réseau social mondial où 10232 personnages historiques débattent de tout, de la tolérance religieuse aux droits des animaux, de la vulcanologie à l'archéologie classique, de la modélisation économique à la culture des célébrités. ${ }^{5}$ Malgré leur étendue, ces deux projets - qui ne sont que deux exemples parmi d'autres, choisis de part et d'autre de la Manche, reposent sur l'exploitation de corpus associés en tout premier lieu aux savants et à ceux que l'on n'appelle pas encore les intellectuels, c'està-dire à des représentants issus majoritairement d'une certaine couche de la société, presque tous hommes, membres d'une élite souvent urbaine.

3 L'ensemble des travaux réunis ici permet de nuancer le tableau: il montre que la diffusion des savoirs passe aussi par des canaux plus inattendus que les institutions savantes, que les femmes y jouent un rôle non négligeable. La transmission emprunte, surtout pour s'immiscer dans le quotidien, des routes diverses, dont certaines paraissent aux antipodes de l'érudition, s'appuyant sur des innovations pédagogiques et visant des publics parfois fort éloignés des cercles raffinés de la haute culture ${ }^{6}$. La période des Lumières constitue un moment central pour réfléchir sur cette question et constitue à certains égards un laboratoire expérimental.

4 Un petit retour sur le vocabulaire permet de rappeler deux idées centrales présentes dans les termes choisis par différentes cultures européennes pour désigner les Lumières. Certaines langues mettent en évidence un processus comparable à un éveil, comme l'allemand avec l'Aufklärung ou l'anglais avec l'Enlightenment. Les idiomes latins omettent cet aspect-là mais soulignent une forme de multiplicité, voire de prolifération, ce sont I Lumi, c'est el Siglo de las Luces, le Siècle des Lumières ou les Lumières tout court. Action engagée et expressions multiples caractérisent ainsi les Lumières, comprises comme un ensemble de pratiques sociales et de systèmes de communication au cœur duquel appréhender la question de la circulation des savoirs et 
de l'importance des projets éducatifs. Un ouvrage récent résume cette unité dans la diversité qui constitue une ligne de force centrale :

Ce qui caractérise les Lumières comme mouvement intellectuel, ce qui en constitue l'unité au-delà des divergences théoriques, c'est la dimension militante et pédagogique, la conviction que la lutte contre les préjugés et les superstitions doit être menée publiquement, que le savoir et l'esprit critique doivent être diffusés le plus largement possible. ${ }^{7}$

Si les circulations de manuscrits considérés comme dangereux sont de mieux en mieux connues, notamment grâce aux travaux développés autour de La Lettre clandestine, et celles des savoirs et des idées mises en évidence dans différents ouvrages ${ }^{8}$, la question de la pédagogie et celle du rôle des femmes ont longtemps été des parents pauvres dans le domaine9. Or, si l'on se penche sur les textes et les contextes, mais aussi sur les acteurs et publics de cette grande entreprise pédagogique à la fois de plus en plus diversifiée et démocratique, force est de constater l'intérêt porté à l'éducation du public féminin et au rôle que les femmes elles-mêmes ont joué ${ }^{10}$.

6 L'une des figures phares, en France comme au-delà de l'Hexagone, est sans aucun doute Marie Leprince de Beaumont (1711-1780 ?). Si Sarah Fielding ${ }^{11}$, avec The Governess, or The Little Female Academy (1749) lui dame le pion en inventant la fiction pédagogique dialoguée à l'usage des maîtresses et de leurs élèves, elle ne connaîtra pas le succès de son imitatrice normande. La formule de Leprince de Beaumont, très proche de celle de l'Anglaise, profite sans doute de l'importance du français comme langue véhiculaire à l'époque. Le Magasin des enfants (1756) et la série d'autres publications conçues dans le même esprit comptent parmi les ouvrages les plus traduits et diffusés en Europe. Ils sont lus dans différents milieux, y compris les plus exaltés - les travaux de Jill Shefrin mettent par exemple en évidence l'importance de ces œuvres pédagogiques ${ }^{12}$ jusqu'à la Cour. Ils reposent en partie sur des strates de savoirs, ainsi que sur ce que Jeanne Chiron appelle "[une] Dynamique de retranscription, [des] jeux de mise en fiction. ${ }^{13}$ Polygraphe, romancière, journaliste et pédagogue, Leprince de Beaumont pose la question des Lumières comme phénomène démocratique, paneuropéen et pluriel ${ }^{14}$. L'ensemble de son œuvre illustre sa foi dans le progrès et dans la perfectibilité de l'homme et elle participe activement à une entreprise de diffusion du savoir dont l'encyclopédisme constitue l'un des versants. De même, elle défend le droit de la femme à l'égalité intellectuelle et à un savoir parfois genré. Elle s'interroge sur le problème de la hiérarchie sociale, sur les enjeux du mariage ou sur des questions comme la liberté. Marie Leprince de Beaumont s'inscrit ainsi pleinement dans les débats intellectuels et religieux de son siècle. Elle a cependant traditionnellement été associée plutôt aux Anti-Lumières en raison du caractère apologétique de ses ouvrages qui ont conduit nombre de chercheurs à les laisser de côté et à ne pas percevoir les aspects novateurs de celle qui entend faire, selon ses propres termes, des "philosophes» de fillettes de cinq ans confiées à sa charge.

7 Leprince de Beaumont n'est pas la seule à s'intéresser à la manière dont son sexe peut contribuer au progrès. D'autres comme Félicité de Genlis ou Louise d'Épinay démontrent le rôle des femmes dans la diffusion des savoirs au siècle des Lumières.

Malgré l'intérêt croissant porté à ces questions ces dernières années, nous sommes loin d'avoir répondu à toutes les interrogations concernant les textes mêmes, leurs contextes, la nature des savoirs diffusés, les acteurs et leurs publics, les voies et modalités de circulation ainsi que la question des médias et des mises en scène 
(textuelles) des processus de diffusion et/ou d'enseignement. Divers aspects moins connus de la diffusion des savoirs sont abordés dans ce numéro.

L'article d'Hélène Boons porte sur la transformation du genre du « Spectateur » dans le Nouveau Magasin français, journal dirigé par Marie Leprince de Beaumont durant les années $1750-1752^{15}$. Comme en d'autres occasions, l'écrivaine née à Rouen s'approprie des modèles textuels et littéraires, en l'occurrence ici, ceux d'Addison et de Marivaux, qu'elle transforme à ses propres fins éducatives. Dans ce cas précis, il s'agit de concevoir un spectateur adolescent, et en partie aussi féminin, ce qui invite à des réflexions en matière de méthodologie pédagogique, à un moment où s'intensifie le regard sur cette période critique d'évolution et de maturation de l'individu. Les réécritures de Leprince de Beaumont attestent par ailleurs les processus de transferts culturels, par la réception et la circulation des textes à échelle transnationale à l'époque des Lumières. Nicolas Brucker relève des phénomènes analogues en se penchant sur l'enseignement de la Bible dans les Magasins de Leprince de Beaumont : la formation religieuse proposée peut sembler peu orthodoxe à bien des égards, mais elle obéit au double impératif de trouver une méthodologie adéquate pour enseigner la Bible à un public enfantin, et de former, au passage, une pensée critique ${ }^{16}$. Les récits bibliques tels que les transmet Marie Leprince de Beaumont sont marqués par le protestantisme (horresco referens pour une femme comme elle) qui influe ainsi (clandestinement) sur l'herméneutique proposée.

Abordant l'usage des contes et des fables chez les auteurs-éducateurs de la seconde moitié du XVIII ${ }^{\mathrm{e}}$ siècle, Aurélia Gaillard se concentre également sur des questions de méthodologie pédagogique. Il s'agit d'une part de faire passer une morale de manière compréhensible (et de déjouer ainsi la critique que Rousseau exprime contre la fable dans l'Émile), et, de l'autre, de communiquer «l'envie de l'histoire », donc de la lecture aux enfants. Avec une perspective ouverte à d'autres éducateurs et éducatrices, Aurélia Gaillard évoque également les conditions de genèse d'une partie des textes qui s'inspirent souvent de l'activité pédagogique de leurs auteur.e.s.

11 Si Marie Leprince de Beaumont fut gouvernante de jeunes filles nobles à Londres, Félicité de Genlis, à laquelle est dédiée l'intervention de Marie-Emmanuelle Plagnol, fut gouverneur des enfants du duc d'Orléans ${ }^{17}$. Elle assumait un rôle à contre-emploi en termes de genre - et le titre au masculin, qu'elle exigea de porter, le met clairement en évidence. Les manœuvres de Genlis sont de mieux en mieux connues, grâce entre autres à la publication de documents restés longtemps inédits ${ }^{18}$. Montrant les strates de textes qui servent à la fois à recenser les leçons données et apprises et à exercer un contrôle sur les élèves du gouverneur, Philippe Lejeune propose de parler du «Panoptique de Mme de Genlis $»^{19}$. L'apport de Marie-Emmanuelle Plagnol dans le présent numéro est de relever les traces de manipulations effectuées par la pédagogue au sein de ses Mémoires. Le but est clair : d'offrir au lecteur une vision cohérente $a$ posteriori de son projet éducatif. Modalités de composition de ses textes, évolution de sa posture d'auteure, tout est bon pour celle qui entend corriger son image et en renforcer les aspects édifiants. À l'instar de Leprince de Beaumont, Genlis inscrit son œuvre dans un cadre référentiel consciemment catholique - et parfois militant - ce qui impose de reconnaître une fois de plus ce que l'historiographie française a longtemps voulu nier : l'existence de Lumières catholiques.

12 Un autre pédagogue qui s'affiche à l'occasion comme à l'écoute des mouvements et tendances, Louis-Antoine Caraccioli, a souffert d'un dédain critique analogue à celui 
accordé à Leprince de Beaumont ou à Genlis. Comme elles, il est associé, par son adhésion au catholicisme, à des courants réactionnaires. Comme elles encore, c'est un polygraphe. Comme elles, c'est un auteur de textes éducatifs qui a connu une expérience pédagogique concluante: l'auteur du Véritable Mentor (1756) a servi de précepteur à de jeunes nobles polonais. Stéphanie Géhanne Gavoty se penche sur un volume dans lequel une forme goûtée du public et une apparence d'authenticité sont mises au service d'une hagiographie à double détente ${ }^{20}$. Il s'agit d'un côté de louer un pape défunt, Clément XIV, en traçant des épisodes de sa vie par ce recueil, et de conduire le lecteur à en adopter les idées. Au passage, Ganganelli est rendu philosophe et la religion devient affaire de bon sens pour un auditoire étendu. La forme elle-même évolue avec une place accordée à des documents, à des réponses, à l'illustration d'une actualité des idées du pontife défunt. En réalité, comme le démontre l'article, dans cette " fraude pieuse ", Caraccioli, pour lequel « l'éducation est l'art des arts " ${ }^{21}$, prête à celui qui ne peut désormais pas s'en défendre, ses propres idées pédagogiques. Il les enrobe ainsi d'une autorité beaucoup moins contestable que la sienne.

13 Si Caraccioli a besoin, pour se rendre crédible, d'endosser les oripeaux du pape, Malesherbes, au contraire, dispose d'une autorité qui ira croissant au cours de sa vie au point d'en faire une figure centrale de l'État. Réformateur zélé, mu par un sens du bien collectif, il se donne pour but d'améliorer le sort de ses contemporains. Ainsi que le montre Valérie André, un aspect méconnu de son entreprise le voit prendre à bras-lecorps le système éducatif ${ }^{22}$. Nous ne sommes plus dans le rapport direct de précepteur ou de gouvernante à élève, comme dans la pratique de Leprince de Beaumont, Genlis ou Caraccioli, ni dans la diffusion générale d'idées dont on ne sait quel retentissement elles auront auprès des lecteurs putatifs, par le biais des ouvrages pédagogiques, mais bien dans une notion de service public et de normalisation. Le projet s'inscrit dans une actualité : le Parlement de Paris réagit aux conséquences de l'expulsion des jésuites à la fin de novembre 1764. Celle-ci rend en effet critique une réflexion sur le devenir possible d'un certain nombre d'institutions éducatives désormais dépossédées de leurs enseignants. Le lecteur pourra s'en étonner, le projet d'une éducation nationale, prôné peu d'années plus tard à l'issue de la Révolution sous le titre d'« instruction publique », n'emporte pas l'adhésion de Malesherbes : il ne veut pas de l'uniformisation. Il entend cependant qu'une administration spécifiquement dévolue à la question se charge de l'éducation des Français, pas un Parlement qui ferait mal la tâche. Comme d'autres idées promues par le réformateur, celle-ci resta dans les cartons. Elle esquisse pourtant, dans certaines de ses lignes, des perspectives que l'avenir devait voir mises en place.

Si les observations de Malesherbes n'ont guère circulé, le contraire est vrai des vers de Jacques Delille. Véritable bestseller des Lumières, son Homme des champs offre au lecteur, dans l'esprit virgilien qui caractérise nombre d'œuvres du «dupeur d'oreilles», aussi bien une expérience littéraire agréable qu'un mode d'emploi. Ainsi que le montre Hugues Marchal, Delille est à la fois élève et maitre ${ }^{23}$ : il diffuse dans son poème des connaissances acquises à l'occasion d'entretiens avec des scientifiques et le fait paraître rehaussé de notes dont il délègue la responsabilité au savant Hermann. Le partage des voix pourrait donner une autorité particulière à l'appareil critique, traditionnellement dévolu à la précision et aux détails là où le vers pouvait se permettre, pour provoquer un effet frappant, de présenter une vision impressionniste. Or, le nom du naturaliste n'est pas utilisé comme argument de vente supplémentaire, ni comme légitimation du contenu de l'édition. Une enquête dans les manuscrits permet de mettre en évidence une réalité complexe: Hermann est certes un collaborateur de Delille mais il a 
l'impression de n'avoir pas vu son expertise reconnue à sa juste valeur et il devient, ainsi que le montre l'article, un adversaire actif du projet de l'homme de lettres, visant, au-delà d'un dialogue entre poésie et science typique de maints érudits des Lumières, une dimension expérimentale que le texte descriptif peine à rendre. À trop vouloir réconcilier des contraires, ne risque-t-on pas d'en lisser les caractéristiques?

Un dernier texte nous conduit du côté d'un contemporain de Delille qui met en évidence les aspérités plutôt que de les gommer. On sait que «les instituteurs immoraux » sont à l'œuvre dans le boudoir et que le château de Silling accueille "l'école du libertinage ». Marco Menin met en évidence un travail de sape continuel chez Sade ${ }^{24}$. C'est parce qu'il connaît les ressorts et la rhétorique de la pédagogie rousseauiste que le divin marquis peut les déjouer de l'intérieur. Les libertins des Liaisons dangereuses de Laclos suscitent notre admiration. Ceux de la fiction de Sade, et en particulier de ses contes trop négligés, provoquent parfois la sympathie du lecteur, ébahi par leur intelligence. Tout en paraissant rejeter le pathos, l'écrivain le détourne en le dé-moralisant et en fait le fondement d'une esthétique au cœur de ce que l'on pourrait comprendre comme un projet anthropologique. Il s'agit ainsi, comme dans la fiction édifiante d'une Leprince de Beaumont, de manipuler le lecteur et de le conduire à adhérer à une doctrine. Si celle de La Philosophie dans le boudoir est aux antipodes de celle du Magasin des adolescentes, les deux dialogues ne s'attachent pas moins à transmettre des savoirs à des jeunes femmes de la bonne société.

Les textes, les individus et les idées se complètent, s'entrechoquent et vont parfois jusqu'à se contredire. Les Lumières plurielles ne sont donc pas univoques, bien au contraire. À côté des grands noms retenus par l'Histoire de la littérature ou celle des mouvements d'idées, nombre d'écrivains - dont certains comme Delille ou Leprince de Beaumont furent célèbres en leur temps - diffusent des notions centrales autour de questions comme le progrès, l'égalité ou la formation de l'esprit critique. Ce sont eux dont les livres sont loués dans les cabinets de lecture ou se retrouvent dans les catalogues de toutes les bibliothèques publiques et privées de leur époque. L'absence de morgue, la volonté de démocratiser les savoirs, le souhait d'engager un dialogue - entre individus, entre domaines d'étude, entre textes et traditions - conduit ces auteurs dits souvent de second rang à mettre de côté un savoir souvent très pointu pour l'accommoder à un lectorat étendu. En cela, ce sont les tâcherons des Lumières, certes, mais les tâcherons ont sans doute plus fait pour la diffusion d'un certain nombre d'idées centrales que les grands noms cités dans les pages des manuels.

\section{NOTES}

1. Nous avons appris, au moment de préparer ce texte, la disparition prématurée de Sophie Lefay qui suivait nos travaux et dont la finesse d'analyse, la modestie érudite et la générosité rayonnante nous manqueront.

2. Germaine de Staël, De l'Allemagne, 1813. Voir L'idée de l'Europe au Siècle des Lumières, éd. de Rotraud von Kulessa et Catriona Seth, Cambridge, Open Book, 2017, p. 126, accessible en ligne : 
https://www.openbookpublishers.com/reader/610\#page/140/mode/2up, consulté le 13 mai 2020.

3. http://enccre.academie-sciences.fr/encyclopedie/, consulté le 13 mai 2020.

4. https://www.e-enlightenment.com/, consulté le 13 mai 2020.

5. "Listen in on the first global social network as 10,232 historical figures discuss everything from religious tolerance to animal rights, vulcanology to classical archeology, economic modelling to celebrity culture.", Ibid.

6. Le projet EDULUM (Éducatrices des Lumières : le cas de Marie Leprince de Beaumont), financé par l'ANR et la DFG (2015-2018), et que nous avons codirigé, et dont le colloque final a inclus parmi ses présentations celles qui sont regroupées ici, s'est évertué à examiner certains aspects de cette large question.

7. Antoine Lilti, L'héritage des Lumières. Ambivalences de la modernité, Paris, Seuil/Gallimard, 2019, p. 270.

8. Daniel Roche, Humeurs vagabondes. De la circulation des hommes et de l'utilité des voyages, Paris, Fayard, 2003 ; Pierre-Yves Beaurepaire (dir.), La communication en Europe. De l'âge classique au siècle des Lumières, Paris, Belin, 2014.

9. Denise Escarpit, La littérature d'enfance et de jeunesse en Europe. Panorama historique, Paris, PUF, 1981 ; Rotraud von Kulessa (dir.), Démocratisation et diversification. Les littératures d'éducation au siècle des Lumières, Paris, Classiques Garnier, 2015. Voir aussi le projet en cours d'Alicia C. Montoya, Mediate accessible via http://bibliomediator.nl/, consulté le 14 mai 2020.

10. Sonnet, Martine, L'éducation des filles au temps des Lumières, Paris, Éd. du Cerf, 1987 ; Isabelle Brouard-Arends et Marie-Emmanuelle Plagnol-Diéval (dir.), Femmes éducatrices au Siècle des Lumières, Rennes, PUR, 2007.

11. Voir entre autres Avery, Gillian, "Written for Children: Two Eighteenth-Century English Fairy Tales”, Marvels \& Tales, vol. XVI, n² 2, 2002, p. 143-155. En ligne : www.jstor.org/stable/41388624, consulté le 13 mai 2020.

12. Voir Shefrin, Jill, Such Constant Affectionate Care. Lady Charlotte Finch - Royal Governess \& the Children of George III, Los Angeles, The Cotsen Occasional Press, 2003.

13. Voir Chiron, Jeanne, "Dynamique de retranscription, jeux de mise en fiction » dans Jeanne Chiron et Catriona Seth (dir.), Marie Leprince de Beaumont. De l'éducation des filles à «La Belle et la Bête », Paris, Classiques Garnier, 2013, p. 85-102.

14. Voir sur Leprince de Beaumont les deux volumes issus des rencontres et travaux dans le cadre d'EDULUM : Jeanne Chiron et Catriona Seth (dir.), Marie Leprince de Beaumont. De l'éducation des filles à "La Belle et la Bête », op. cit. et Kulessa, Rotraud von et Seth, Catriona (dir.), Une éducatrice des Lumières, Marie Leprince de Beaumont, Paris, Classiques Garnier, 2018. Voir aussi Herz-Gazeau, Ramona, La Femme entre raison et religion Les Américaines (1769) de Marie Leprince de Beaumont, Paris, Classiques Garnier, 2019 et l'exposition en ligne http://leprincedebeaumont.univ-lorraine.fr/fr, consulté le 13 mai 2020.

15. «Mme Leprince de Beaumont, nouvelle Spectatrice? Parcours adolescents entre Addison et Marivaux ».

16. « La Bible en Magasin. Bible et pédagogie chez Leprince de Beaumont ».

17. «Les Mémoires de Mme de Genlis : un regard rétrospectif sur ses ouvrages éducatifs ».

18. Voir en particulier Gardeur-Lebrun, Charles et Orléans, Louis-Philippe de, Journaux de voyage et d'éducation, Spa, été 1787, éd. de Isabelle Havelange, Paris, Classiques Garnier, 2015 et Bonnard, Bernard de, Journal de l'éducation des princes d'Orléans, décembre 1777-janvier 1782, éd. Dominique Julia, Paris, Classiques Garnier, 2019.

19. Voir Lejeune, Philippe, "Le Panoptique de Mme de Genlis», Le Temps des femmes. Textes mémoriels des Lumières, éd. Anne Coudreuse et Catriona Seth, Paris, Classiques Garnier, 2014, p. 45-68.

20. « Direction et éducation genrée dans Les Lettres du pape Clément XIV de Caraccioli » 
21. Caraccioli, Louis-Antoine de, Le Véritable Mentor, ou l'éducation de la noblesse, par le marquis Caraccioli, colonel au service du roi de Pologne, électeur de Saxe, Liège, Bassompierre ; Francfort, en foire ; Bruxelles, J. Vanden Berghen, 1759, p. XII.

22. «Le libéralisme à l'école. Malesherbes et la réforme des études (1780-1787) ».

23. «Feux conjugués ou opposés : le dialogue entre lettres et sciences à la lueur des notes de Hermann pour L'Homme des champs de Delille ».

24. "Sade éducateur : perversion textuelle et transmission de savoirs dans l'œuvre romanesque sadienne ».

\section{AUTEURS}

\section{CATRIONA SETH}

University of Oxford

ROTRAUD VON KULESSA

Universität Augsburg 